\title{
ON WAVE MOTION IN AN INFINITE SOLID BOUNDED INTERNALLY BY A CYLINDER OR A SPHERE
}

\author{
ARNOLD N. LOWAN
}

\section{PART I}

In two previous papers, $\uparrow$ the author investigated the problem of wave motion for infinite domains of one, two, and three dimensions and for certain sub-infinite domains; that is, domains bounded in certain directions but extending to infinity in other directions. The present paper is a sequel to the aforementioned papers and deals with the problem of wave motion in an infinite solid, bounded internally by a cylinder or a sphere.

In the subsequent developments we shall use the following abbreviations:

$$
\sigma(\alpha)=\left(a^{2} \alpha^{2}-k^{2}\right)^{1 / 2}, \quad s(p, \alpha)=\alpha^{2}+\left(p^{2}-k^{2}\right) / a^{2},
$$

where $\alpha$ is a real variable ranging from $-\infty$ to $\infty$ and $p$ is a complex variable whose real part is positive. We shall also introduce the operators $\nabla_{c}, \nabla_{s}, \sum \iiint$, and $\sum \iiint \int$ defined as follows:

$$
\begin{gathered}
\nabla_{c}=\frac{\partial^{2}}{\partial r^{2}}+\frac{1}{r} \frac{\partial}{\partial r}-\frac{1}{a^{2}}\left(p^{2}-k^{2}\right)+\frac{1}{r^{2}} \frac{\partial^{2}}{\partial \theta^{2}} \\
\nabla_{s}=\frac{\partial^{2}}{\partial r^{2}}+\frac{2}{r} \frac{\partial}{\partial r}+\frac{1}{r^{2} \sin \theta} \frac{\partial}{\partial \theta}\left(\sin \theta \frac{\partial}{\partial \theta}\right)+\frac{1}{r^{2} \sin ^{2} \theta} \frac{\partial^{2}}{\partial \phi^{2}} \\
-\frac{1}{a^{2}}\left(p^{2}-k^{2}\right), \\
\sum \iiint\left\{F_{n}\left(r^{\prime}, \theta^{\prime}, \alpha\right)\right\}=\sum_{n=0}^{\infty}(2 n+1) \cos n\left(\theta-\theta^{\prime}\right) \int_{R}^{\infty} r^{\prime} d r^{\prime} \\
\sum \iiint \int\left\{F_{n}\left(r^{\prime}, \theta^{\prime}, \phi^{\prime}, \alpha\right)=\sum_{n=0}^{2 \pi}(2 n+1) P_{n}^{\infty}(\cos \gamma)\right. \\
\cdot \int_{R}^{\infty} r^{\prime 3 / 2} d r_{n}\left(r^{\prime}, \theta^{\prime}, \alpha\right) d \alpha \\
\sum \sin \theta^{\prime} d \theta^{\prime} \int_{0}^{2 \pi} d \phi^{\prime} \int_{-\infty}^{\infty} \alpha F_{n}\left(r^{\prime}, \theta^{\prime}, \phi^{\prime}, \alpha\right) d \alpha,
\end{gathered}
$$

$\dagger$ On wave motion for infinite domains, Philosophical Magazine, (7), vol. 26 (1938), pp. 340-360; On wave motion for sub-infinite domains, Philosophical Magazine, (7), vol. 27 (1939), pp. 182-194. These papers will be referred to as L-1 and L-2, respectively. 
where $P_{n}$ is the Legendre polynomial of degree $n$ and $\gamma$ is the angle between the vectors from the origin to the points $(r, \theta, \phi)$ and $\left(r^{\prime}, \theta^{\prime}, \phi^{\prime}\right)$.

Consider first the case of an infinite solid bounded internally by a cylinder. In this case the displacement satisfies the system A consisting of equations (1), (2), (3), and (4):

$$
\begin{array}{rlrl}
\nabla^{2} U(P ; t) & =2 b \frac{\partial}{\partial t} U(P ; t)+\frac{1}{a^{2}} \frac{\partial^{2}}{\partial t^{2}} U(P ; t)+\Phi(P ; t), \\
\lim _{t \rightarrow 0} U(P ; t) & =f(P), & \\
\lim _{t \rightarrow 0} \frac{\partial}{\partial t} U(P ; t) & =g(P), & r=R .
\end{array}
$$

As in L-1 and L-2, we put

$$
U(P ; t)=u(P ; t)+v(P ; t),
$$

where $u(P ; t)$ satisfies the system $\mathrm{B}$, consisting of (1), (2), (3), and the boundary condition

$$
u(P ; t)=0, \quad r=R,
$$

and where $v(P ; t)$ satisfies the system $\mathrm{C}$, obtained from $\mathrm{A}$, by replacing $U(P ; t)$ by $v(P ; t)$ and putting $f(P)=g(P)=\Phi(P ; t)=0$. We proceed to the solution of the systems $\mathrm{B}$ and $\mathrm{C}$.

As in L-1 and L-2, the method of solving the systems B and C consists in making the substitution

$$
\begin{aligned}
& u(r, \theta ; t)=e^{-k t} u_{1}(r, \theta ; t), \\
& \Phi(r, \theta ; t)=e^{-k t} \Phi_{1}(r, \theta ; t), \\
& v(r, \theta ; t)=e^{-k t} v_{1}(r, \theta ; t),
\end{aligned}
$$

where $k=b a^{2}$.

Let $\mathrm{B}_{1}$ and $\mathrm{C}_{1}$ designate the systems obtained from the systems $\mathrm{B}$ and $C$, by the substitutions (7), (8), and (9) for the functions $u_{1}, v_{1}$, and $\Phi_{1}$. The solutions of the last two systems are obtained by operating on systems $\mathrm{B}$ and $\mathrm{C}$ with the Laplace operator and obtaining the systems $\mathrm{B}_{1}^{*}$ and $\mathrm{C}_{1}^{*}$, for the Laplace transforms $u_{1}^{*}(r, \theta ; p)$ and $v_{1}^{*}(r, \theta ; p)$. When the solutions of $\mathrm{B}_{1}^{*}$ and $\mathrm{C}_{1}^{*}$ have been obtained, the corresponding solutions of $B$ and $C$ are obtained by acting on the corresponding solutions with the inverse Laplace operator. The system $\mathrm{B}_{1}^{*}$ is ultimately obtained in the following form: 


$$
\begin{aligned}
\nabla_{c} u_{1}^{*}(r, \theta ; p) & =-\frac{1}{a^{2}}\{p f(r, \theta)+h(r, \theta)\}+\Phi_{1}^{*}(r, \theta ; p) \\
& =-F(r, \theta ; p)(\mathrm{say}) \\
h(r, \theta) & =g(r, \theta)+k f(r, \theta) \\
u_{1}^{*}(R, \theta ; p) & =0
\end{aligned}
$$

and the system $\mathrm{C}_{1}^{*}$ in the form

$$
\begin{aligned}
\nabla c v_{1}^{*}(r, \theta ; p) & =0, \\
v_{1}^{*}(R, \theta ; p) & =\phi_{1}^{*}(\theta ; p) .
\end{aligned}
$$

In order to obtain the solution of $\mathrm{B}_{1}{ }^{*}$, we make use of the identity $\dagger$

$$
f(r, \theta)=\frac{1}{4 \pi} \sum \iiint\left\{f\left(r^{\prime}, \theta^{\prime}\right) G_{n}\left(r, r^{\prime} ; \alpha\right)\right\}
$$

where

$$
G_{n}\left(r, r^{\prime} ; \alpha\right)=\frac{H_{n}^{1}(\alpha r)}{H_{n}^{1}(\alpha R)}\left\{J_{n}\left(\alpha r^{\prime}\right) \cdot H_{n}^{1}(\alpha R)-J_{n}(\alpha R) H_{n}^{1}\left(\alpha r^{\prime}\right)\right\}
$$

for $r^{\prime}<r$; the corresponding expression for $r^{\prime}>r$, is obtained by interchanging $r$ and $r^{\prime}$.

In view of (14), it can be verified that the expression

$$
u_{1}^{*}(r, \theta ; p)=\frac{1}{4 \pi} \sum \iiint\left\{F\left(r^{\prime}, \theta^{\prime} ; p\right) \cdot \frac{G_{n}\left(r, r^{\prime} ; \alpha\right)}{s(p, \alpha)}\right\}
$$

is the solution of the system $B_{1}{ }^{*}$.

Bearing in mind the significance of $F(r, \theta ; p)$ from $(10),(15)$ becomes

$$
u_{1}^{*}(r, \theta ; p)=u_{1,1}^{*}(r, \theta ; p)+u_{1,2}^{*}(r, \theta ; p)+u_{1,3}^{*}(r, \theta ; p)
$$

where

$$
\begin{aligned}
& u_{1,1}^{*}(r, \theta ; p)=\frac{1}{4 \pi a^{2}} \sum \iiint\left\{f\left(r^{\prime}, \theta^{\prime}\right) \frac{p G_{n}\left(r, r^{\prime} ; \alpha\right)}{s(p, \alpha)}\right\} \\
& u_{1,2}^{*}(r, \theta ; p)=\frac{1}{4 \pi a^{2}} \sum \iiint\left\{h\left(r^{\prime}, \theta^{\prime}\right) \frac{G_{n}\left(r, r^{\prime} ; \alpha\right)}{s(p, \alpha)}\right\} \\
& u_{1,3}^{*}(r, \theta ; p)=-\frac{1}{4 \pi} \sum \iiint\left\{G_{n}\left(r, r^{\prime} ; \alpha\right) \frac{\Phi_{1}^{*}\left(r^{\prime}, \theta^{\prime} ; p\right)}{s(p, \alpha)}\right\}
\end{aligned}
$$

$\dagger$ See Appendix at end of this paper, §1. 
The problem now reduces to obtaining the inverse Laplace transforms of (17), (18), and (19). Making use of the identities

$$
\int_{0}^{\infty} e^{-p t} \cos \lambda t d t=\frac{p}{p^{2}+\lambda^{2}}, \quad \int_{0}^{\infty} e^{-p t} \sin \lambda t d t=\frac{\lambda}{p^{2}+\lambda^{2}},
$$

and of Borel's theorem, we finally get

$$
\begin{aligned}
& u_{1,1}(r, \theta ; t)=\frac{1}{4 \pi} \sum \iiint\left\{f\left(r^{\prime}, \theta^{\prime}\right) G_{n}\left(r, r^{\prime} ; \alpha\right) \cos \sigma(\alpha) t\right\} \\
& u_{1,2}(r, \theta ; t)=\frac{1}{4 \pi} \sum \iiint\left\{h\left(r^{\prime}, \theta^{\prime}\right) G_{n}\left(r, r^{\prime} ; \alpha\right) \frac{\sin \sigma(\alpha) t}{\sigma(\alpha)}\right\}, \\
& u_{1,3}(r, \theta ; t) \\
& =-\frac{a^{2}}{4 \pi} \int_{0}^{t} \sum \iiint\left\{\Phi_{1}\left(r^{\prime}, \theta^{\prime} ; t-\tau\right) G_{n}\left(r, r^{\prime} ; \alpha\right) \frac{\sin \sigma(\alpha) \tau}{\sigma(\alpha)}\right\} d \tau .
\end{aligned}
$$

In the case where the solutions $f, g, \phi$, and $\Phi$ do not depend on $\theta$, it is clear that the final solution is also independent of $\theta$. In this case, it can be shown (see Appendix, §2) that the identity (14) must be replaced by

$$
f(r)=\frac{1}{4 \pi} \int_{R}^{\infty} r^{\prime} d r^{\prime} \int_{-\infty}^{\infty} \alpha f\left(r^{\prime}\right) G_{0}\left(r, r^{\prime} ; \alpha\right) d \alpha,
$$

where the expression for $G_{0}$ is obtained from that for $G_{n}$, by replacing the index $n$ by zero.

In view of this result, the solutions for $u_{1,1}, u_{1,2}$, and $u_{1,3}$, when these solutions do not depend on $\theta$, may be obtained at once from (20), (21), and (22), by dropping the summation sign and the factor $\cos n\left(\theta-\theta^{\prime}\right)$ and replacing the subscript $n$ by zero.

We proceed to the solution of the system $\mathrm{C}_{1}{ }^{*}$. The expression

$$
v_{1}^{*}(r, \theta ; p)=\frac{1}{\pi} \sum_{n=-\infty}^{\infty} \int_{0}^{2 \pi} \phi_{1}^{*}\left(\theta^{\prime} ; p\right) \cos n\left(\theta-\theta^{\prime}\right) w_{n}^{*}(r ; p) d \theta^{\prime}
$$

where

$$
w_{n}^{*}(r ; p)=\frac{H_{n}^{1}(\alpha r)}{H_{n}^{1}(\alpha R)} \text { (say), }
$$

is a solution of (12), satisfying the boundary conditions (13), provided

$$
s(p, \alpha)=0 \text {. }
$$

If the function $w_{n}(r, t)$ is defined by 


$$
\int_{0}^{\infty} e^{-p t} w_{n}(r ; t) d t=\frac{H_{n}^{1}(\alpha r)}{H_{n}^{1}(\alpha R)}=w_{n}^{*}(r ; p) \text { (say), }
$$

then by Borel's theorem, the inverse Laplace transform of (23) is

$$
v_{1}(r, \theta ; t)=\frac{1}{\pi} \sum_{-\infty}^{\infty} \int_{0}^{2 \pi} \int_{0}^{t} \phi_{1}\left(\theta^{\prime} ; t-\tau\right) w_{n}(r, \tau) \cos n\left(\theta-\theta^{\prime}\right) d \theta^{\prime} d \tau .
$$

Our problem thus reduces to solving the integral equation (26), where $\alpha$ is defined in (25). Since $H_{n}{ }^{1}(z) \rightarrow 0$ in the upper half of the $z$-plane and since its zeros are known to lie in the lower half of the plane, it can be easily shown with the aid of the Cauchy integral theorem that

$$
\begin{aligned}
w_{n}^{*}(r ; p) & =\frac{H_{n}^{1}(r \alpha)}{H_{n}^{1}(R \alpha)}=\frac{1}{\pi i} \int_{-\infty}^{\infty} \frac{x}{x^{2}-\alpha^{2}} \cdot \frac{H_{n}^{1}(r x)}{H_{n}^{1}(R x)} d x \\
& =\frac{a^{2}}{\pi i} \int_{-\infty}^{\infty} \frac{x}{p^{2}+[\sigma(x)]^{2}} \cdot \frac{H_{n}^{1}(r x)}{H_{n}^{1}(R x)} d x .
\end{aligned}
$$

The inversion of (26) leads at once to

$$
w_{n}(r ; t)=\frac{a^{2}}{\pi i} \int_{-\infty}^{\infty} \frac{x \sin \sigma(x) t}{\sigma(x)} \frac{H_{n}^{1}(r x)}{H_{n}^{1}(R x)} d x .
$$

In (27) and (29), we have the complete solution of the system $B_{1}$.

It should be remarked that the expression $w_{n}(r ; t)$ given by (29) is real. Indeed, if in the contribution to the integral in (29) for the range from $-\infty$ to 0 we make the substitution $x=-\xi$, replace once more the variable of integration $\xi$ by $x$, and furthermore, make use of the well known relation

$$
H_{n}^{1}(-z)=(-1)^{n}\left[H_{n}^{1}(z)-2 J_{n}(z)\right],
$$

(29) becomes

$$
\begin{aligned}
w_{n}(r ; t)=\frac{a^{2}}{\pi i} \int_{0}^{\infty} \frac{x \sin \sigma(x) t}{\sigma(x)}\left\{\frac{H_{n}^{1}(r x)}{H_{n}^{1}(R x)}\right. & \\
& \left.-\frac{H_{n}^{1}(r x)-2 J_{n}(r x)}{H_{n}^{1}(R x)-2 J_{n}(R x)}\right\} d x .
\end{aligned}
$$

Since $H_{n}^{1}(z)=J_{n}(z)+i Y_{n}(z)$, the above equation ultimately becomes

$$
w_{n}(r ; t)=\frac{2 a^{2}}{\pi} \int_{0}^{\infty} \frac{x \sin \sigma(x) t}{\sigma(x)} \cdot \frac{J_{n}(R x) Y_{n}(r x)-J_{n}(r x) Y_{n}(R x)}{\left(J_{n}(R x)\right)^{2}+\left(Y_{n}(R x)\right)^{2}} d x .
$$

If the function $\phi$ is independent of $\theta$, it is clear that $v_{1}$ is independ- 
ent of $\theta$. In this case, instead of (23), we start with

$$
v_{1}^{*}(r ; p)=\phi_{1}^{*}(p) w_{0}^{*}(r ; p),
$$

where $w_{0}^{*}(r ; p)$ may be obtained from $(28)$ by replacing the subscript $n$ by zero. The corresponding expression for $w_{0}(r, t)$ may therefore be obtained from (29) by replacing the subscript $n$ by zero. With this definition of $w_{0}(r ; t)$ we have

$$
v_{1}(r ; t)=\int_{0}^{\infty} \phi_{1}(t-\tau) w_{0}(r ; \tau) d \tau
$$

\section{PART II. CASE OF AN INFINITE SOlID BOUNDED INTERNALLY BY A SPHERE}

In this case, the displacement $U(P ; t)$ must satisfy a system similar to $\mathrm{A}$, except that $\nabla^{2} u(P ; t)$ is now the Laplacian in spherical coordi nates and the boundary condition (4) may assume the more general form

$$
U(P ; t)=\Omega(\theta, \phi ; t), \quad r=R .
$$

The method of solution is entirely similar to that in Part I. The Laplace transforms of the functions $u_{1}(P ; t)$ and $v_{1}(P ; t)$ obtained from $u(P ; t), v(P ; t)$ by the substitutions $(7),(8)$, and $(9)$, for the case under consideration, must now satisfy the systems $D_{1}^{*}$, consisting of equations (33) and (34), and $\mathrm{E}_{1}^{*}$, consisting of (35) and (36):

$$
\begin{aligned}
\nabla_{s} u_{1}^{*}(r, \theta, \phi ; p) & =-\frac{1}{a^{2}}\{p f(r, \theta, \phi)+h(r, \theta, \phi)\}+\Phi_{1}^{*}(r, \theta, \phi ; p) \\
& =-F(r, \theta, \phi ; p)(\mathrm{say}), \\
u_{1}^{*}(R, \theta, \phi ; p) & =0, \\
\nabla_{s} v_{1}^{*}(r, \theta, \phi ; p) & =0, \\
v_{1}^{*}(r, \theta, \phi ; p) & =\Omega_{1}^{*}(\theta, \phi ; p) .
\end{aligned}
$$

We proceed to the solution of the system $\mathrm{D}_{1}^{*}$. In this case, we make use of the following identity; $\dagger$

$$
f(r, \theta, \phi)=\frac{1}{8 \pi r^{1 / 2}} \sum \iiint \int\left\{f\left(r^{\prime}, \theta^{\prime}, \phi^{\prime}\right) G_{n+1 / 2}\left(r, r^{\prime}, \alpha\right)\right\} .
$$

As in Part I, it can be verified that the expression

$\dagger$ The derivation of this identity is discussed in the Appendix, $\$ 4$. The expression for $G_{n+1 / 2}\left(r, r^{\prime}, \alpha\right)$ may be obtained from that of $G_{n}\left(r, r^{\prime}, \alpha\right)$ by replacing the subscript $n$ by $n+1 / 2$. 
(38) $u_{1}^{*}(r, \theta, \phi ; p)=\frac{1}{8 \pi r^{1 / 2}} \sum \iiint \int\left\{F\left(r^{\prime}, \theta^{\prime}, \phi^{\prime}\right) \frac{G_{n+1 / 2}\left(r, r^{\prime}, \alpha\right)}{s(p, \alpha)}\right\}$

is a solution of the system $D_{1}^{*}$, provided that the expression for $G_{n+1 / 2}\left(r, r^{\prime}, \alpha\right)$ in (38) is obtained from the expression $G_{n}\left(r, r^{\prime}, \alpha\right)$ by replacing the subscript $n$ by $n+1 / 2$. The expressions for $u_{1,1}, u_{1,2}$, and $u_{1,3}$ may therefore be obtained at once from the corresponding expressions in Part $\mathrm{I}$ in the forms

$$
\begin{gathered}
u_{1,1}(r, \theta, \phi ; t)=\frac{1}{8 \pi r^{1 / 2}} \sum \iiint \int\left\{\begin{array}{c}
f\left(r^{\prime}, \theta^{\prime}, \phi^{\prime}\right) \\
\left.\cdot G_{n+1 / 2}\left(r, r^{\prime} ; \alpha\right) \cos \sigma(\alpha) t\right\}
\end{array}\right. \\
u_{1,2}(r, \theta, \phi ; t)=\frac{1}{8 \pi r^{1 / 2}} \sum \iiint \int\left\{h\left(r^{\prime}, \theta^{\prime}, \phi^{\prime}\right)\right. \\
\left.\cdot G_{n+1 / 2}\left(r, r^{\prime} ; \alpha\right) \frac{\sin \sigma(\alpha) t}{\sigma(\alpha)}\right\}, \\
u_{1,3}(r, \theta, \phi ; t)=-\frac{a^{2}}{8 \pi r^{1 / 2}} \int_{0}^{t} \sum \iiint \int\left\{\Phi_{1}\left(r^{\prime}, \theta^{\prime}, \phi^{\prime} ; t-\tau\right)\right. \\
\left.\cdot G_{n+1 / 2}\left(r, r^{\prime} ; \alpha\right) \frac{\sin \sigma(\alpha) \tau}{\sigma(\alpha)}\right\} d \tau .
\end{gathered}
$$

We now proceed to the solution of the system $\mathrm{E}_{1}^{*}$. The expression

$$
\begin{array}{r}
v_{1}^{*}(r, \theta, \phi ; p)=\sum_{n=0}^{\infty} \int_{0}^{\pi} \int_{0}^{2 \pi}(2 n+1) P_{n}(\cos \gamma) \\
\cdot \frac{H_{n+1 / 2}^{1}(\alpha r)}{H_{n+1 / 2}^{1}(\alpha R)} \Omega_{1}^{*}\left(\theta^{\prime}, \phi^{\prime} ; p\right) \sin \theta^{\prime} d \theta^{\prime} d \phi^{\prime}
\end{array}
$$

is a solution of $\mathrm{E}_{1}^{*}$, provided

$$
s(p, \alpha)=0
$$

(see Appendix, §3).

If, then, the function $w_{n+1 / 2}(r, t)$ is defined by

$$
\int_{0}^{\infty} e^{-p t} w_{n+1 / 2}(r ; t) d t=\frac{H_{n+1 / 2}^{1}(\alpha r)}{H_{n+1 / 2}^{1}(\alpha R)}=w_{n+1 / 2}^{*}(r ; p) \text { (say), }
$$

then by Borel's theorem, the inversion of (42) yields

$$
\begin{aligned}
v_{1}(r, \theta, \phi ; t)=\sum_{n=0}^{\infty} \int_{0}^{\pi} \int_{0}^{2 \pi} \int_{0}^{t}(2 n+1) P_{n}(\cos \gamma) \\
\cdot \Omega_{1}\left(\theta^{\prime}, \phi^{\prime} ; t-\tau\right) w_{n+1 / 2}\left(r^{\prime}, \tau\right) d \theta^{\prime} d \phi^{\prime} d \tau .
\end{aligned}
$$


Our problem thus reduces to solving the integral equation (44).

This equation is identical with (26) except that the subscript $n$ is replaced by $n+1 / 2$. The method of solution of (45) is entirely similar to that of (26), and we finally get

$$
w_{n+1 / 2}(r ; t)=\frac{a^{2}}{\pi i} \int_{-\infty}^{\infty} \frac{x \sin \sigma(x) t}{\sigma(x)} \cdot \frac{H_{n+1 / 2}^{1}(r x)}{H_{n+1 / 2}^{1}(R x)} d x,
$$

or (by a transformation similar to that for (29))

$$
\begin{aligned}
w_{n+1 / 2}(r ; t)=\frac{2 a^{2}}{\pi} & \int_{0}^{\infty} \frac{x \sin \sigma(x) t}{\sigma(x)} \\
& \frac{J_{n+1 / 2}(R x) Y_{n+1 / 2}(r x)-J_{n+1 / 2}(r x) Y_{n+1 / 2}(R x)}{\left(J_{n+1 / 2}(R x)\right)^{2}+\left(Y_{n+1 / 2}(R x)\right)^{2}} d x .
\end{aligned}
$$

The complete solution in the case of an infinite solid bounded internally by a sphere is therefore given by

$$
U(P ; t)=e^{-b a^{2} t}\left(u_{1,1}+u_{1,2}+u_{1,3}+v\right),
$$

where the terms in parentheses are given by (39), (40), (41), and (45).

An important special case is that in which the functions $f, g, \Omega, \Phi$ do not depend on the angles $\theta$ and $\phi$. While the solution can be obtained from the previous solution by integrating the variables $\theta^{\prime}$ and $\phi^{\prime}$, it is easier to proceed as follows. We have to solve the system of equations

$$
\begin{aligned}
\frac{\partial^{2} u}{\partial r^{2}}+\frac{2}{r} \frac{\partial u}{\partial r} & =2 b \frac{\partial u}{\partial t}+\frac{1}{a^{2}} \frac{\partial^{2} u}{\partial t^{2}}+\Phi(r ; t), & & R<r<\infty, \\
\lim _{t \rightarrow 0} u(r ; t) & =f(r), & & R<r<\infty, \\
\lim _{t \rightarrow 0} \frac{\partial}{\partial t} u(r ; t) & =g(r), & & R<r<\infty,
\end{aligned}
$$

and

$$
U(R ; t)=\phi(t) .
$$

If we make the substitution $u(r ; t)=(1 / r) v(r ; t)$, it is readily seen that the function $v(r ; t)$ must satisfy the system

$$
\begin{aligned}
\frac{\partial^{2} v}{\partial r^{2}} & =2 b \frac{\partial v}{\partial t}+\frac{1}{a^{2}} \frac{\partial^{2} v}{\partial t^{2}}+r \Phi(r ; t), \\
\lim _{t \rightarrow 0} v(r ; t) & =r f(r),
\end{aligned}
$$




$$
\begin{aligned}
\lim _{t \rightarrow 0} \frac{\partial}{\partial t} v(r ; t) & =r g(r), \\
V(R ; t) & =R \phi(t) .
\end{aligned}
$$

The system satisfied by $v(r, t)$ is formally identical with that corresponding to wave motion in a semi-infinite solid extending from $r=R$ to $r=\infty$.

The solution corresponding to a solid extending from 0 to $\infty$ is given in L-2. It is clear that our present solution may be obtained by replacing $x$ by $r-R$, in L-2.

\section{Appendix}

1. Derivation of the identity (14). Consider the problem of heat conduction in an infinite solid, bounded internally by a cylinder, the surface of which is kept at $0^{\circ}$. The solution of this problem may be obtained with the aid of the appropriate Green's function for a point source. The expression for the Green's function is

$$
G\left(r, \theta ; t ; r^{\prime}, \theta^{\prime}\right)=\frac{1}{4 \pi} \sum_{-\infty}^{\infty} \cos n\left(\theta-\theta^{\prime}\right) \int_{-\infty}^{\infty} \alpha e^{-k \alpha^{2} t}
$$

for $r<r^{\prime}$.

$$
\frac{H_{n}^{1}\left(\alpha r^{\prime}\right)}{H_{n}^{1}(\alpha R)}\left\{J_{n}(\alpha r) H_{n}^{1}(\alpha R)-J_{n}(\alpha R) H_{n}^{1}(\alpha r)\right\} d \alpha
$$

In the case $r>r^{\prime}$, the corresponding expression may be obtained by interchanging $r$ and $r^{\prime}$. With the aid of the general formulas of Carslaw's $\dagger$ article 80 , the solution of the problem of heat conduction under consideration is seen to be

$$
T(r, \theta ; t)=\frac{1}{4 \pi} \sum \iiint\left\{f\left(r^{\prime}, \theta^{\prime}\right) P_{n}(\cos \gamma) \cdot e^{-k \alpha^{2} t} G_{n}\left(r, r^{\prime}, \alpha\right)\right\}
$$

Putting $t=0$, we obtain the identity (14).

2. Derivation of identity $\left(14^{\prime}\right)$. The expression for the Green's function corresponding to a cylindrical source may be obtained by considering a continuous distribution of line sources around the cylinder $r=r^{\prime}$, integrating for the variable $\theta^{\prime}$ and dividing by $2 \pi$.

The corresponding solution of the problem in heat conduction can then be obtained from (II) by dropping the summation sign and the factor $\cos n\left(\theta-\theta^{\prime}\right)$ and replacing the subscript $n$ by zero. If in this final solution we make $t=0$, we obtain the identity $\left(14^{\prime}\right)$.

\footnotetext{
$\dagger$ Carslaw, Mathematical Theory of Heat Conduction.
} 
3. Derivation of identity (42). Starting with the well known expansion

$$
\begin{aligned}
& F(\theta, \phi)= \sum_{n=0}^{\infty} \sum_{m=-n}^{n} \int_{0}^{\pi} \int_{0}^{2 \pi} \frac{(n-|m|) !}{(n+|m|) !} \\
& \cdot F\left(\theta^{\prime}, \phi^{\prime}\right) P_{n}^{m}(\cos \theta) P_{n}^{m}\left(\cos \theta^{\prime}\right) e^{i m}\left(\phi-\phi^{\prime}\right) \sin \theta^{\prime} d \theta^{\prime} d \phi^{\prime}
\end{aligned}
$$

where the $P_{n}{ }^{m}$ 's are the associated Legendre polynomials, and making use of the identity (see Carslaw's article 93)

$$
\begin{aligned}
P_{n}(\cos \gamma)=P_{n}(\cos \theta) P_{n}\left(\cos \theta^{\prime}\right)+2 \sum_{m=1}^{\infty} & \frac{(n-|m|) !}{(n+|m|) !} P_{n}^{m}(\cos \theta) \\
& \cdot P_{n}^{m}\left(\cos \theta^{\prime}\right) \cos m\left(\phi-\phi^{\prime}\right),
\end{aligned}
$$

we find that the second member of (42) reduces to $\Omega_{1}^{*}(\theta, \phi ; p)$ for $r=R$.

4. Derivation of identity (37). The Green's function corresponding to a point source in an infinite solid bounded internally by a sphere, the surface of which is kept at $0^{\circ}$, is given by

$$
\begin{aligned}
G\left(r, \theta, \phi ; t ; r_{0}, \theta_{0},\right. & \left.\phi_{0}\right)=\frac{1}{8 \pi\left(r r_{0}\right)^{1 / 2}} \sum_{n=0}^{\infty}(2 n+1) P_{n}(\cos \gamma) \\
& \cdot \int_{-\infty}^{\infty} \alpha e^{-k \alpha^{2} t} \frac{H_{n+1 / 2}^{1}\left(\alpha r_{0}\right)}{H_{n+1 / 2}^{1}(\alpha R)} \\
& \cdot\left\{J_{n}(\alpha r) H_{n+1 / 2}^{1}(\alpha R)-J_{n+1 / 2}(\alpha R) H_{n+1 / 2}^{1}(\alpha r)\right\} d \alpha .
\end{aligned}
$$

With the aid of the general formula of Carslaw's article 80, the solution of the appropriate problem in heat conduction is found to be

$$
T(r, \theta, \phi ; t)=\frac{1}{8 \pi r^{1 / 2}} \sum \iiint \int\left\{e^{-k \alpha^{2} t} f\left(r^{\prime}, \theta^{\prime}, \phi^{\prime}\right) G_{n+1 / 2}\left(r, r^{\prime}\right)\right\}
$$

Putting $t=0$, we obtain identity (37).

Brooklyn College AND

Yeshiva College 